Research Article

\title{
Potential Genes Associated with the Survival of Lung Adenocarcinoma Were Identified by Methylation
}

\author{
Ziyuan Shen, ${ }^{1}$ Chenlu He, ${ }^{1}$ Haimiao Chen, ${ }^{1}$ Lishun Xiao, ${ }^{1,2}$ Yingliang Jin $\mathbb{D D}^{1,2}$ \\ and Shuiping Huang $\mathbb{D}^{1,2}$ \\ ${ }^{1}$ Department of Epidemiology and Biostatistics, School of Public Health, Xuzhou Medical University, Xuzhou, Jiangsu 221004, China \\ ${ }^{2}$ Center for Medical Statistics and Data Analysis, School of Public Health, Xuzhou Medical University, Xuzhou, \\ Jiangsu 221004, China
}

Correspondence should be addressed to Yingliang Jin; yljin@xzhmu.edu.cn and Shuiping Huang; hsp@xzhmu.edu.cn

Received 10 July 2020; Revised 19 August 2020; Accepted 27 October 2020; Published 18 November 2020

Academic Editor: Emil Alexov

Copyright ( 2020 Ziyuan Shen et al. This is an open access article distributed under the Creative Commons Attribution License, which permits unrestricted use, distribution, and reproduction in any medium, provided the original work is properly cited.

\begin{abstract}
Background. Lung adenocarcinoma (LUAD) is the most common pathological type of lung cancer. The purpose of this study is to search for genes related to the prognosis of LUAD through methylation based on a linear mixed model (LMM). Methods. Gene expression, methylation, and survival data of LUAD patients were downloaded from the TCGA database. Based on the LMM model, the GEMMA algorithm was used to screen the predictive genes related to LUAD survival. The Cox model was used to further screen the predicted genes, and then, protein-protein interaction (PPI) network was constructed. Through the software plugin Cytoscape MCODE 3.8.0, the most closely related genes in the PPI network module were selected for in-depth biological function analysis to further explore the interaction and correlation between genes. Results. We screened out 97 predictive genes from 18,834 genes and eliminated one gene associated with lung squamous cell carcinoma from previous studies, leaving 96 genes. The MCODE and the Kaplan-Meier curve analysis were used to finally identify two genes ASB16 and NEDD4 that are related to the prognosis of LUAD. Conclusions. The newly identified two genes associated with the prognosis of LUAD may provide a basis for the treatment of patients.
\end{abstract}

\section{Introduction}

Global cancer data show that the incidence and mortality rates of lung cancer again top the list [1]. Approximately 520,000 new cases are reported annually in men and 267,000 in women. Nearly $61 \%$ of the pathological subtypes of lung cancer are lung adenocarcinoma (LUAD), and lung cancer poses a serious threat to human health [2]. Pathologically, different types of cancer cells originate from different sites in the lung. LUAD refers to the mucus-secreting epithelial cells that originate from the smaller bronchial mucosa, so most adenocarcinomas are located in the peripheral part of the lung in a spherical mass close to the pleura. Unlike squamous cell lung cancer, LUAD is more likely to occur in women and nonsmokers [3]. However, smoking remains a major environmental risk factor for lung cancer [4]. Causes of high mortality from LUAD include the lack of sensitive and specific early biomarkers, high likelihood of drug resistance, and metastasis [5]. In recent years, some prognostic genes related to LUAD have been found, which provide an effective criterion for early molecular diagnosis of LUAD and greatly promote the treatment of patients. The survival rate of lung cancer is on the rise gradually. In China, the 5-year relative survival rate is about $40.5 \%$. That is up about 10 percent from a decade ago. In this study, the new predictive gene screening model and bioinformatics analysis are used to identify the driver genes associated with LUAD survival and to provide an effective criterion for early molecular diagnosis of LUAD.

Traditional treatments for LUAD usually include surgery, chemotherapy, radiation therapy, and targeted therapy [6]. In the past few years, the research on LUAD has been focused on molecular targeted therapy, controlling the metastasis of LUAD cells, and identifying the target genes [7] regulated by LUAD stem cells. In previous studies, SNP 
was mainly used to predict gene expression, and it has a good performance in predicting gene expression. Previous studies have shown that genes associated with LUAD survival are concentrated in regions such as 5p15.33 and 15q.

Methylation was used to predict gene expression in order to obtain methylation-driven genes associated with LUAD prognosis. DNA methylation is one of the core elements of epigenetic modification and an important signal transduction tool for regulating genome function [4]. In addition, the change of methylation state is an important factor leading to tumor genesis, including the decrease of the methylation level in the whole genome and the abnormal increase of the local methylation level in the $\mathrm{CpG}$ island, which leads to the instability of the genome and the nonexpression of tumor suppressor genes. Therefore, methylation can provide an important basis for early diagnosis and prognosis of cancer and provide a new idea for further clinical application. TCGA is the cancer and tumor gene mapping project initiated by the United States in 2005. The purpose of the project is to study the genome changes in cancer by using genome analysis technology. A large-scale genome sequencing has been done, including more than 30 kinds of cancers. TCGA has laid a foundation for the classification and in-depth study of the molecular pathogenesis of LUAD [8].

To search for genes associated with the prognosis of LUAD, we used an open cancer genome atlas database The Cancer Genome Atlas (TCGA) to obtain genetic and epigenetic data on LUAD [9]. LMM is a multigene model because it assumes that all mutations have a nonzero effect on gene expression. We used the effective GEMMA algorithm to fit the LMM using the limited maximum likelihood method. The gene expression value was predicted by methylation, and predictive genes were screened (defined as genes with $R^{2} \geq 0.05$ ) [10]. The COX model was used to further screen the predictive genes to obtain the genes related to the prognosis of LUAD and to identify the relationship between methylation drive and LUAD. Protein-protein interaction network analysis was performed on these genes to understand the role of methylation in the development and progression of LUAD. The core genes with the highest scores in the highest clusters were extracted by MCODE in Cytoscape software. GO enrichment analysis was performed on the core genes, and Kaplan-Meier curve analysis was drawn.

\section{Methods and Materials}

2.1. Data Processing and Analysis. Gene expression, methylation, and clinical data of LUAD were obtained from UCSC Xena (https://xenabrowser.net/). Samples soaked in formalin-fixed paraffin-embedded tissues were excluded. Quantile conversion was performed by using the qqnorm function in $\mathrm{R}$ software. The original gene expression data included 20,530 genes and 515 samples, and the methylation data came from 458 samples. Firstly, quality control was carried out on the gene expression data, and more than $50 \%$ of the zero expression was eliminated. DNA methylation levels in a group of $500 \mathrm{~kb}$ genes were then filtered by combining gene expression levels with DNA methylation levels. Combining the gene expression and methylation data according
TABLE 1: Basic clinical information of patients with LUAD.

\begin{tabular}{lc}
\hline Clinical parameters & Number of cases \\
\hline Age (years) & \\
$>67$ & 193 \\
$\leq 67$ & 257 \\
Sex & \\
$\quad$ Male & 209 \\
Female & 241 \\
Number-pack-years-smoked & \\
$>37$ & 151 \\
$\leq 37$ & 299 \\
\hline
\end{tabular}

TABLe 2: Abstracts of articles related to LUAD.

\begin{tabular}{lccccc}
\hline PMID & Year & $\begin{array}{c}N \\
\text { (case/control) }\end{array}$ & POP & Genes & Ref \\
\hline 18385676 & 2008 & $1,154 / 1,137$ & European & 6 & {$[11]$} \\
18385738 & 2008 & $1,989 / 2,625$ & European & 5 & {$[12]$} \\
18780872 & 2008 & $194 / 219$ & European & 5 & {$[13]$} \\
18978787 & 2008 & $5,095 / 5,200$ & European & 3 & {$[14]$} \\
18978790 & 2008 & $3,259 / 4,159$ & European & 2 & {$[15]$} \\
19654303 & 2009 & $1.952 / 1,438$ & European & 4 & {$[16]$} \\
19836008 & 2009 & $5,739 / 5,848$ & European & 7 & {$[17]$} \\
20304703 & 2010 & $328 / 407$ & European & 1 & {$[18]$} \\
20700438 & 2010 & $584 / 585$ & East Asian & 2 & {$[19]$} \\
20871597 & 2010 & $1,004 / 1,900$ & Japanese & 2 & {$[20]$} \\
20876614 & 2010 & $1,425 / 3,011$ & Korean & 1 & {$[21]$.} \\
21725308 & 2011 & $2,331 / 3,077$ & Han Chinese & 4 & {$[22]$} \\
21866343 & 2011 & $426 / 497$ & Korean & 3 & {$[23]$} \\
22797724 & 2012 & $1,695 / 5,333$ & Japanese & 4 & {$[24]$} \\
22899653 & 2012 & $14.900 / 29,485$ & European & 1 & {$[25]$} \\
23143601 & 2012 & $5,510 / 4,544$ & East Asian & 9 & {$[26]$} \\
24325914 & 2013 & $2,331 / 3,077$ & Han Chinese & 4 & {$[27]$} \\
24658283 & 2014 & $2,383 / 3,160$ & Han Chinese & 5 & {$[28]$} \\
24880342 & 2014 & $11,348 / 15,361$ & European & 4 & {$[29]$} \\
25145502 & 2014 & 354 & Han Chinese & 1 & {$[30]$} \\
27393504 & 2016 & $1,737 / 3,605$ & African & 6 & {$[31]$} \\
27501781 & 2016 & $663 / 4,367$ & Japerican & & {$[32]$} \\
28604730 & 2017 & $11,273 / 55,483$ & European & 208 & {$[33]$} \\
29924316 & 2018 & $775 / 31,563$ & European & 18 & {$[34]$} \\
30104567 & 2018 & $4,972 / 5,501$ & European & 1 & {$[35]$} \\
31326317 & 2019 & $27,120 / 27,355$ & Han Chinese & 3 & {$[4]$} \\
\hline & & & & &
\end{tabular}

$N$ : initial sample size; POP: population ethnicity.

to the sample name, 18,834 genes and 450 samples were obtained.

A total of 450 samples were included in our analysis, and the clinical variables included age, gender, and annual smoking volume. For details, basic clinical information of patients with LUAD were summarized in Table 1 . The missing values were replaced by the median. 


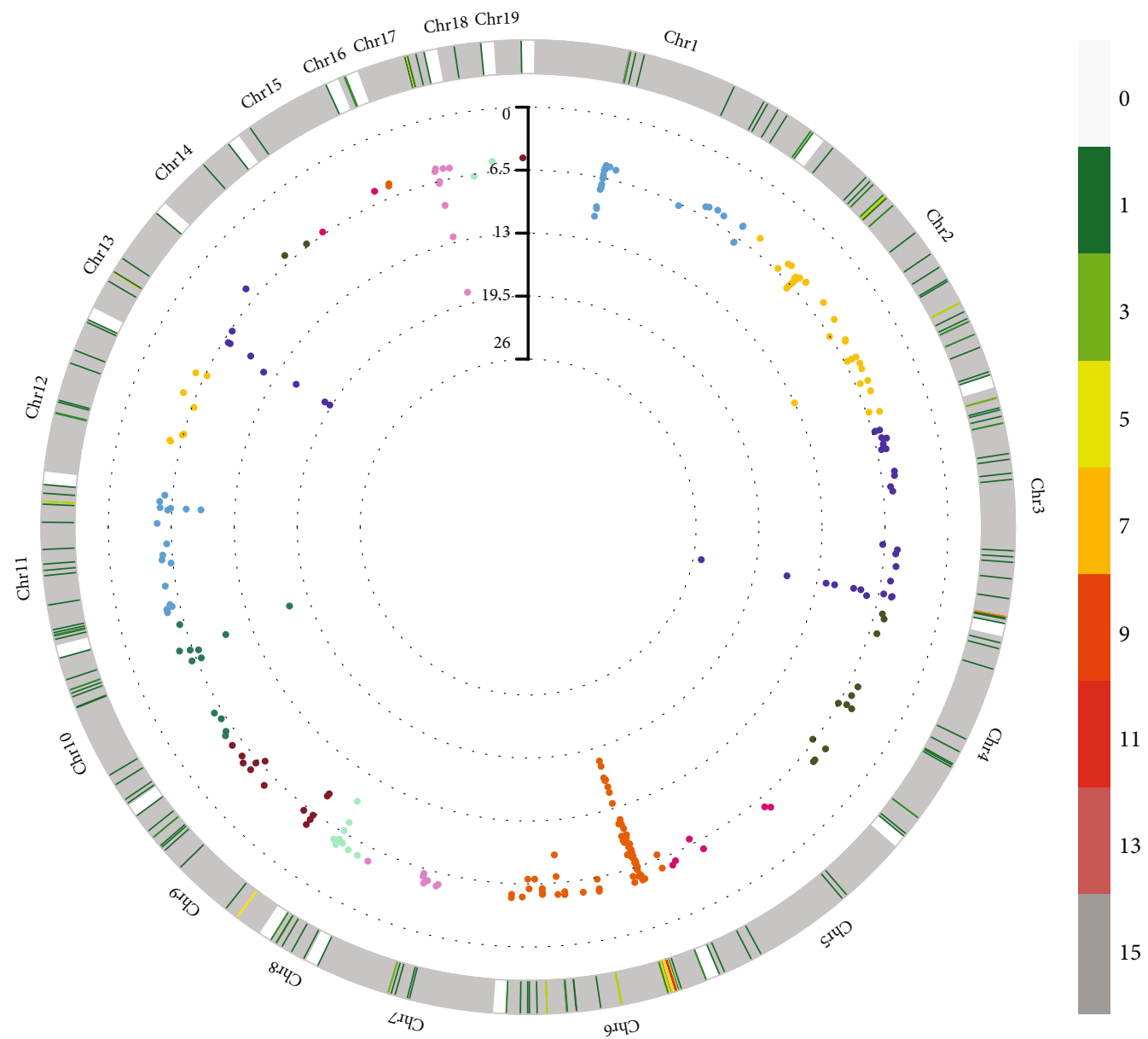

(a)

Figure 1: Continued. 


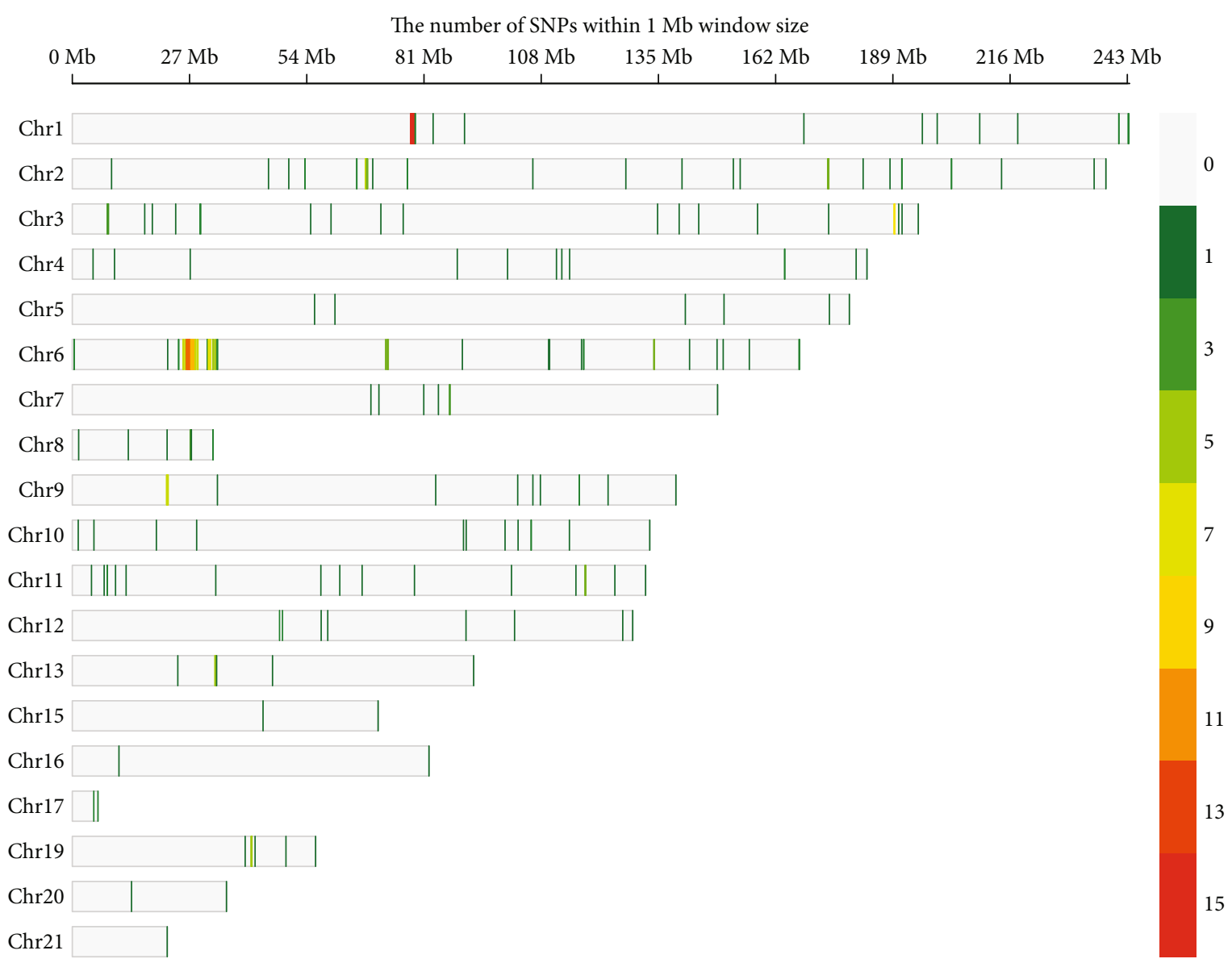

(b)

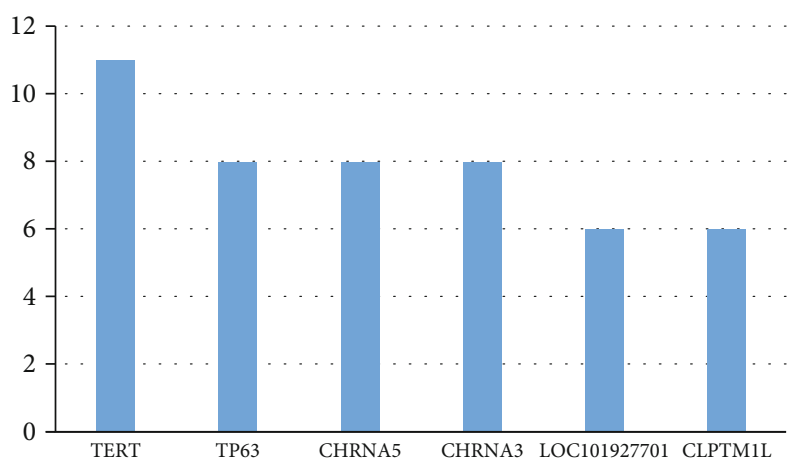

(c)

FIGURE 1: (a) Circular Manhattan diagram of all reported SNPs in GWAS. (b) The number of reported SNPs within 1 Mb window size in GWAS. (c) The most frequently reported genes in these articles.

TABLE 3: Correlation $R^{2}$ values for top ten genes.

\begin{tabular}{lccccc}
\hline Gene & CHR & $R^{2}$ & Gene & CHR & $R^{2}$ \\
\hline FLJ42875 & 1 & 0.904 & FBXL16 & 16 & 0.917 \\
LOC441869 & 1 & 0.931 & MSLN & 16 & 0.952 \\
KCNQ1 & 11 & 0.940 & H3F3B & 17 & 0.923 \\
MUC5B & 11 & 0.903 & ZNF750 & 17 & 0.918 \\
CBFA2T3 & 16 & 0.904 & ADAMTSL5 & 19 & 0.913 \\
\hline
\end{tabular}

2.2. Two-Step Identification of Genes Associated with the Prognosis of LUAD

2.2.1. Predictive Genes Were Identified Using LMM. We bring the data into the linear mixed model. Let us first assume that all the markers are normalized to mean 0 and variance 1 . Let $E_{i}$ be an $n$-vector of the expression level of the $i$ th gene measured on $n$ individuals, $L_{i}$ is the $n \times p$ matrix of DNA methylation. The simple linear model that relates DNA methylation to gene expression level is $E_{i}=L_{i} c_{i}$, where $c_{i}$ is the $p$-vector effect value corresponding to the $i$ th gene. The 


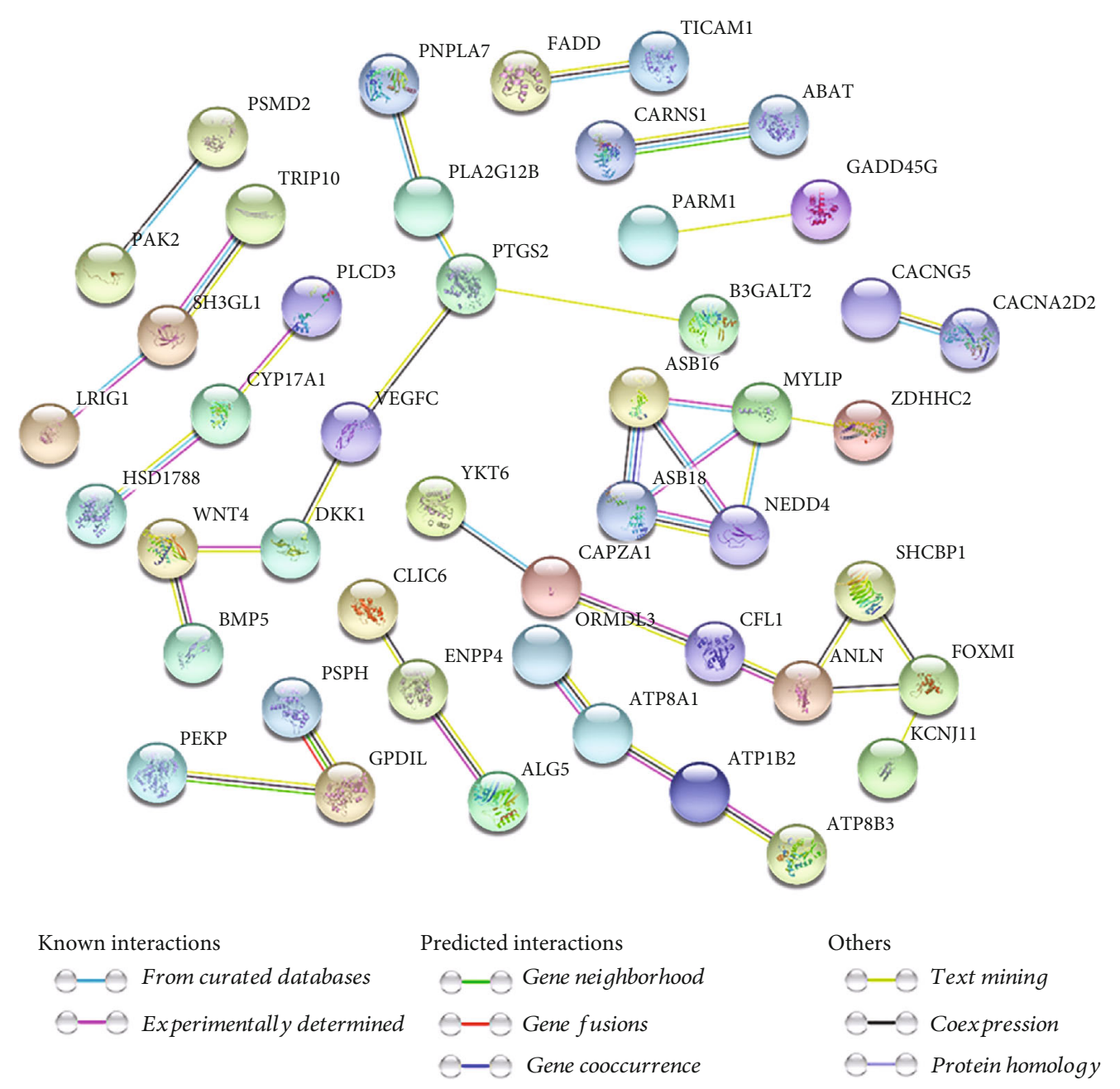

FIGURE 2: Results of protein-protein interaction network analysis.

square correlation coefficient $\left(R^{2}\right)$ of the predicted value is used to measure the performance. The predicted gene expression values can be regarded as the potential effect of DNA methylation. The $R^{2} \geq 0.05$ gene is thought to be methylation driven, and these genes are retained for further analysis.

\subsubsection{Cox Regression Analysis Identified the Prognostic Genes.} The Cox regression model was used to further analyze the predictive genes screened by the linear mixed model and to explore the relationship between methylation-driven genes and the prognosis of LUAD [36]. It is still assumed that all the markers may be involved in the development of LUAD, and the effect size of each gene should follow a normal distribution:

$$
h\left(t_{i} \mid E_{i}, L_{i}\right)=h_{0}\left(t_{i}\right) e^{E_{i}^{T \beta+L_{i}^{T \gamma}}}, \gamma \sim N\left(0, \sigma^{2}\right)
$$

where $h_{0}(t)$ is the arbitrary baseline risk function corresponding to the reference level of the covariates, and $\beta$ is the effect size of gene $i$, and $\gamma=\left(\gamma^{1}, \gamma^{2}, \cdots, \gamma^{m}\right)$ is the $m$ -dimensional vector of the random effect size of DNA methylation; $\sigma^{2}$ is the variance of DNA methylation. We used the false discovery method to adjust the $p$ value results $($ FDR $<0.01)$.

2.3. Protein-Protein Interaction Network and Module Analysis. In order to mine the core regulatory genes, we constructed the protein interaction network by using the STRING database (version 11.0). We also implemented signaling pathways for these genes through Cytoscape software (version 3.8.0) and visualized them through CluePedia. Through the MCODE plugin of Cytoscape software, the most closely connected modules were selected from the constructed PPI network for in-depth biological function analysis [37]. The genes contained in the modules are the core genes.

2.4. Kaplan-Meier Curve Analysis. Kaplan-Meier curve analysis was used to analyze the correlation between core genes and survival. We used the original expression values of genes and the predicted expression values of methylation to calculate their effects on survival, respectively. The prognosis genes were screened with $p<0.05$ as statistically significant difference.

2.5. Gene Set Enrichment Analysis (GSEA). In order to analyze the biological characteristics of prognosis genes and their 


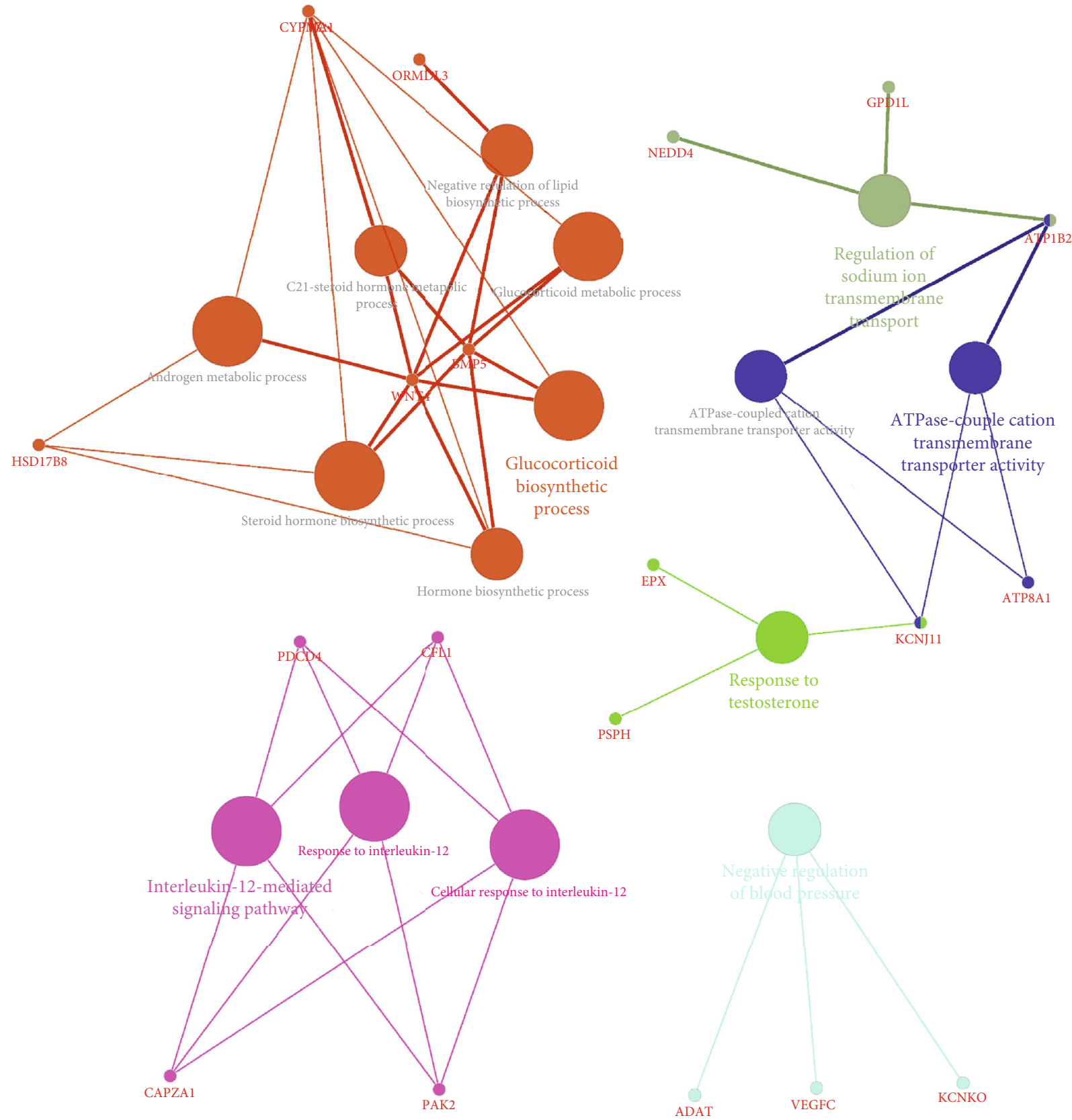

Figure 3: Visualize links between genes. Functionally grouped network with terms as nodes linked based on their kappa score level ( $\geq 0.3)$.

roles in the development of LUAD, the prognosis genes selected by Kaplan-Meier Curve analysis were analyzed by gene set enrichment analysis. GSEA package, clusterProfiler package, and GSEA function were used in $\mathrm{R}$ software to obtain the enrichment results of KEGG pathway and GO pathway, respectively. The number of permutations was set to 1,000 , and a false discovery rate $(\mathrm{FDR})<0.25$ was recognized as statistically significant.

\section{Results}

3.1. Description of Previous Studies. Before October 2019, we searched the GWAS directory with "lung cancer, lung adenocarcinoma" as the search term and conducted a systematic literature search on EBI to preliminarily understand the previous research achievements of LUAD pathogenic genes. A total of 26 articles were included, and these studies were mainly carried out in European populations. Details of the 26 articles we have included are shown in Table 2 and Figure 1, published from 2008 to 2019. A total of 314 genes were reported. The genes associated with LUAD survival were mainly located in 5p15.33, 6p21.3, 15q25, and 17q24.3. By analyzing the GO and KEGG pathways of genes related to LUAD in GWAS, the results showed that gene enrichment molecule functions were mainly identical protein binding, and the biological processes were mainly positive regulation of transcription from RNA polymerase II promoters, and the components mainly included integral 

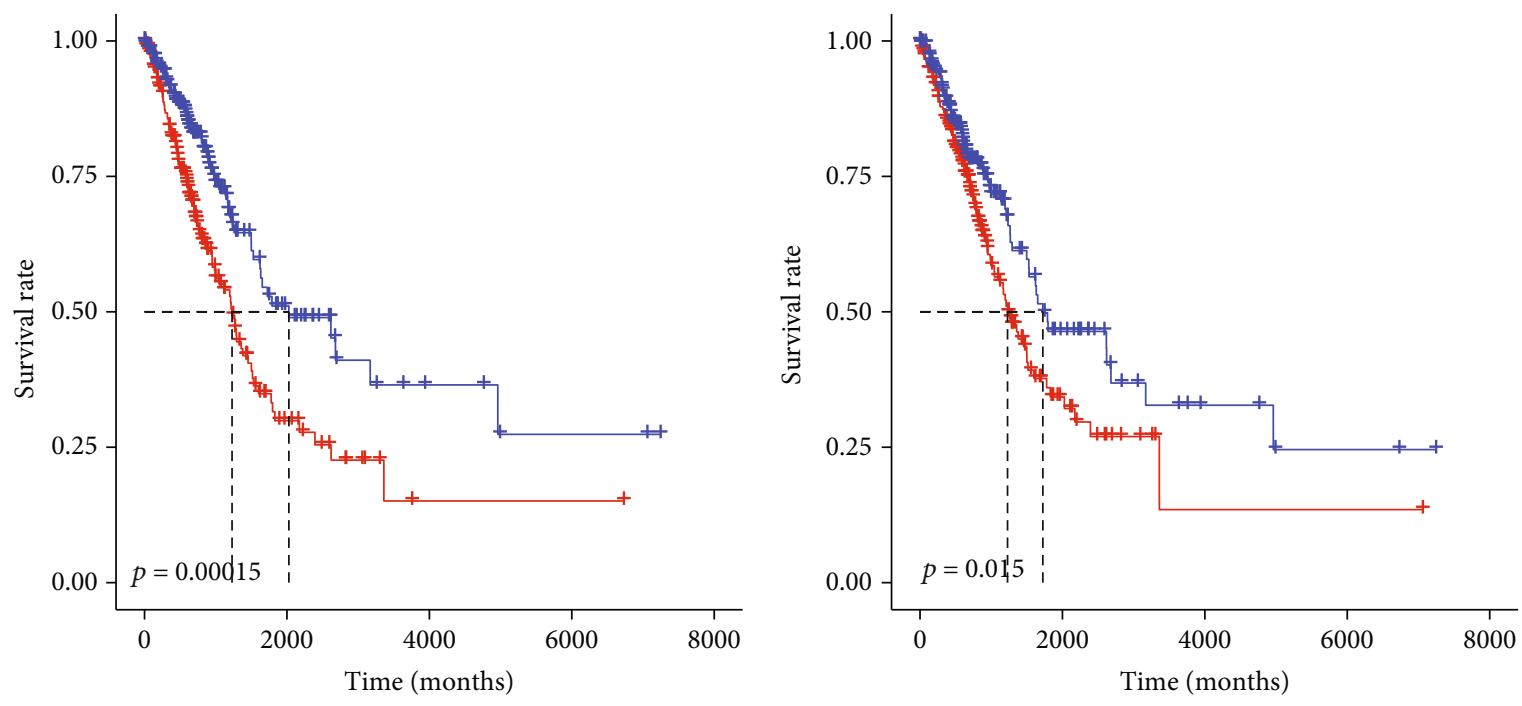

ASB16

+ High risk

ASB16 pred

+ Low risk

(a)

+ High risk

+ Low risk

(b)
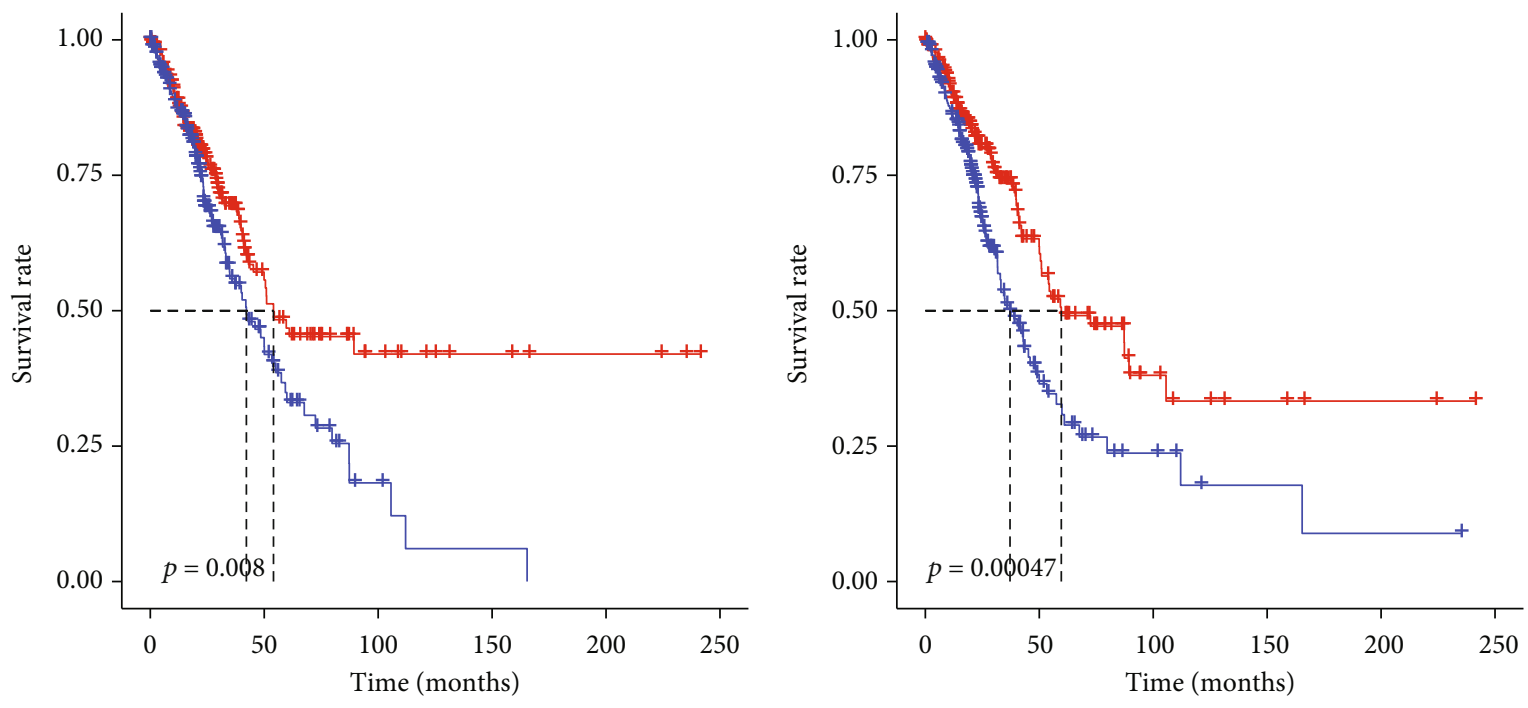

NEDD4

+ High risk

NEDD4 pred

+ High risk

+ Low risk

+ Low risk

(c)

(d)

Figure 4: Continued. 

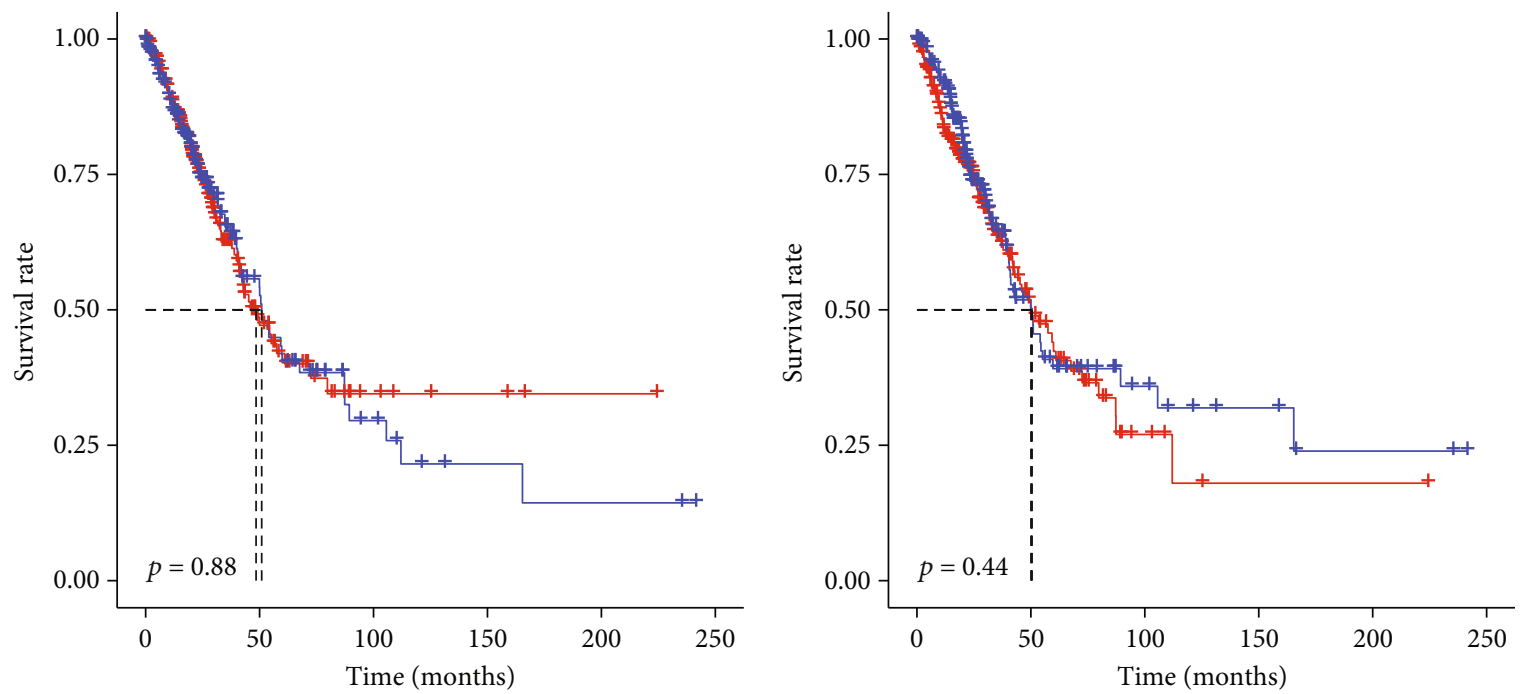

ASB18

ASB18 pred

+ High risk

+ High risk

+ Low risk

+ Low risk

(e)
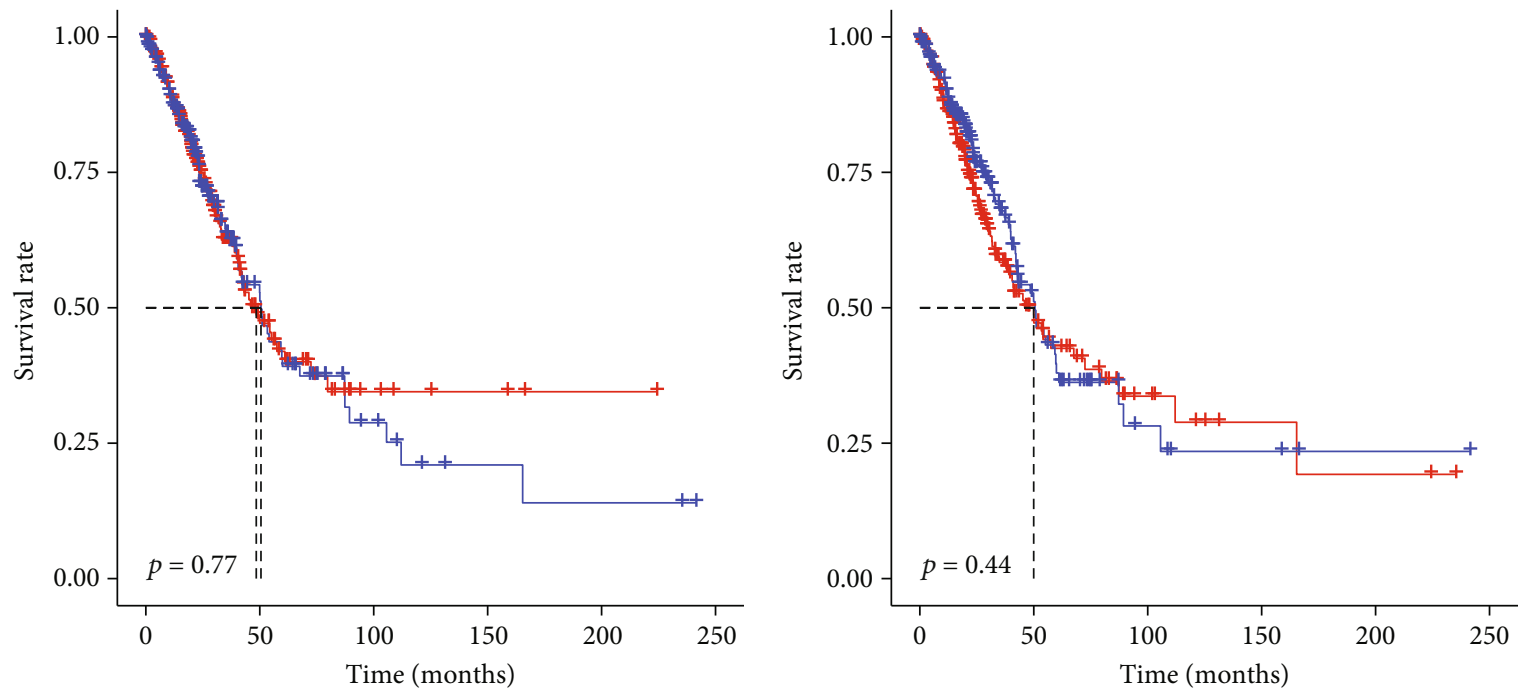

$$
\begin{aligned}
& \text { MYLIP } \\
& + \text { High risk } \\
& + \text { Low risk }
\end{aligned}
$$

(g)

$$
\begin{aligned}
& \text { MYLIP pred } \\
& ++ \text { High risk } \\
& +- \text { Low risk }
\end{aligned}
$$

(h)

FIgURE 4: Kaplan-Meier curve analysis results. (a, b) The combination of gene ASB16 expression and methylation. (c, d) The combination of gene NEDD4 expression and methylation. (e, f) The combination of gene ASB18 expression and methylation. (g, h) The combination of gene MYLIP expression and methylation. pred is the gene expression predicted by methylation.

component of membrane. There were altogether 22 pathways in KEGG. Several articles confirmed that genes TP63, TERT, and CLPTM1L were related to the prognosis of LUAD.

3.2. Results of Linear Mixed Model and Cox Regression Model. After placing 18,834 genes into a linear mixed model, we measured their performance by using the predicted square correlation coefficient $\left(R^{2}\right)$. The results showed that there were 18,495 genes with $R^{2}$ greater than or equal to 0.5 . Table 3 showed information about the ten genes with a higher $R^{2}$ value. A total of 114 prognostic genes were screened by Cox regression model to eliminate the nonprotein-coding genes. Finally, 97 prognostic genes were obtained. After searching on EBI, we excluded DTNBP1, which was linked to lung squamous cell carcinoma in 
TABLE 4: The most significantly enriched signaling pathways.

\begin{tabular}{cccccc}
\hline Gene & MSigDB collection & Gene set name & NES & $p$ val & FDR \\
\hline \multirow{4}{*}{ NEDD4 } & \multirow{4}{*}{ c2.cp.kegg.v7.1.symbols.gmt } & KEGG_REGULATION_OF_ACTIN_CYTOSKELETON & 2.666 & 0.002 & 0.008 \\
& & KEGG_ECM_RECEPTOR_INTERACTION & 2.523 & 0.002 & 0.008 \\
& & c5.bp.v7.1.symbols.gmt & 2.511 & 0.002 & 0.008 \\
& & GO_DRANULOCYTE_MIGRATION & 2.562 & 0.002 & 0.011 \\
& & GO_SUBSTRATE_ADHESION_DEPENDENT_CELL_SPREADING & 2.462 & 0.002 & 0.011 \\
\hline \multirow{4}{*}{ c2.cp.kegg.v7.1.symbols.gmt } & KEGG_TASTE_TRANSDUCTION & 1.701 & 0.002 & 0.088 \\
& & KEGG_ABC_TRANSPORTERS & 1.605 & 0.006 & 0.088 \\
& & KEGG_LINOLEIC_ACID_METABOLISM & 1.518 & 0.031 & 0.139 \\
& & GO_MRNA_SPLICE_SITE_SELECTION & 1.935 & 0.001 & 0.133 \\
& & GO_HISTONE_H3_K27_METHYLATION & 1.893 & 0.001 & 0.133 \\
& & GO_EXTRACELLULAR_TRANSPORT & 1.871 & 0.001 & 0.133 \\
\hline
\end{tabular}

NES: normalized enrichment score; FDR: false discovery rate.

previous studies [38]. In addition, we have identified a smoking-related gene, $A S B 18$, which may further influence the development of lung cancer [39].

3.3. Protein-Protein Interaction Network and Selection of Core Genes. In this study, the protein interaction network was built by using the STRING database (version 11.0). We put 96 genes into STRING, and the species chooses to be Homo sapiens. The PPI score parameter is set at 0.400 (indicating moderate confidence). The network contains 96 nodes and37 edges, and we hide the unconnected nodes in the network. It is worth noting that there is a strong association between the genes of ASB16, ASB18, MYLIP, NEDD4, and ZDHHC2. The result is shown in Figure 2.

Links between genes are visualized through CluePedia, as shown in Figure 3. Through the MCODE plugin of Cytoscape 3.8.0 software (setting parameters as degree cut - off $=$ 2 , node score $=0.2, k$-core $=2$, and maximum depth $=100$ ), the most closely connected modules were selected from the constructed PPI network for in-depth biological function analysis. It was found that the genes included in the most compact modules in the cluster were NEDD4, ASB18, $M Y L I P$, and ASB16, and the highest scoring node in the cluster was $A S B 16$.

3.4. Kaplan-Meier Curve Analysis Results. We used KaplanMeier curves to describe the survival analysis of the four selected genes, and, respectively, analyzed the original gene expression data and the gene expression data predicted by methylation. The results showed that the genes of ASB16 and NEDD4 had a definite effect $(p<0.05)$ on the prognosis of LUAD regardless of the original value or the predictive value, while the genes of ASB18 and MYLIP had no significant effect. The specific results are shown in Figure 4.

3.5. GSEA Results. The GSEA analysis showed that the main functions of the ASB16 gene were covalent chromatin modification, histone methylation, and extracellular transport; the main enrichment pathways were taste transduction, DNA replication, and nucleotide excision repair. The main func- tions of the NEDD4 gene were positive regulation of multiorganism process, regulation of cytoskeleton organization, and divalent inorganic cation homeostasis; the mainly enrichment pathways were MAPK signaling pathway and pathway in cancer. The most significantly enriched signaling pathways based on their NES are shown in Table 4; partial enrichment results are shown in Figure 5.

\section{Discussion}

Lung cancer, as a malignant tumor with high morbidity and mortality in the world, is not only difficult to determine the cause of the disease but also has a poor survival rate. LUAD is the most common pathological classification of lung cancer, so it is of great research value to improve the survival rate of LUAD. The previously identified genes associated with lung cancer and LUAD survival are located mainly on chromosome 6. The enrichment analysis of these genes showed that the molecular function was mainly to selectively and noncovalently interact with the same protein or protein, and the biological process was mainly a process of activating or increasing the transcription frequency, rate, or degree of RNA polymerase II promoter. The component composition mainly included the integral component of the membrane.

In recent years, studies on the survival rate of patients with LUAD have mostly focused on the prediction of genes related to prognosis, the manipulation of the immune system in the treatment of LUAD [6], the study of smoking and the occurrence of LUAD, and the use of SNP to predict the prognosis of LUAD. This study is intended to use the new model to screen the prognostic genes associated with LUAD. The resulrs showed that the two genes were associated with prognosis of LUAD and predictive genes were selected by linear mixed model and Cox regression model. Due to too many screened genes, there was excessive analysis of biological functional analysis of signaling pathways. Therefore, we use the MCODE plugin to connect many genes with a number of genes extracted and then to separate biology related analysis. Gene NEDD4 also enriched in multiple pathways. Previous studies have found that the loci associated with LUAD 


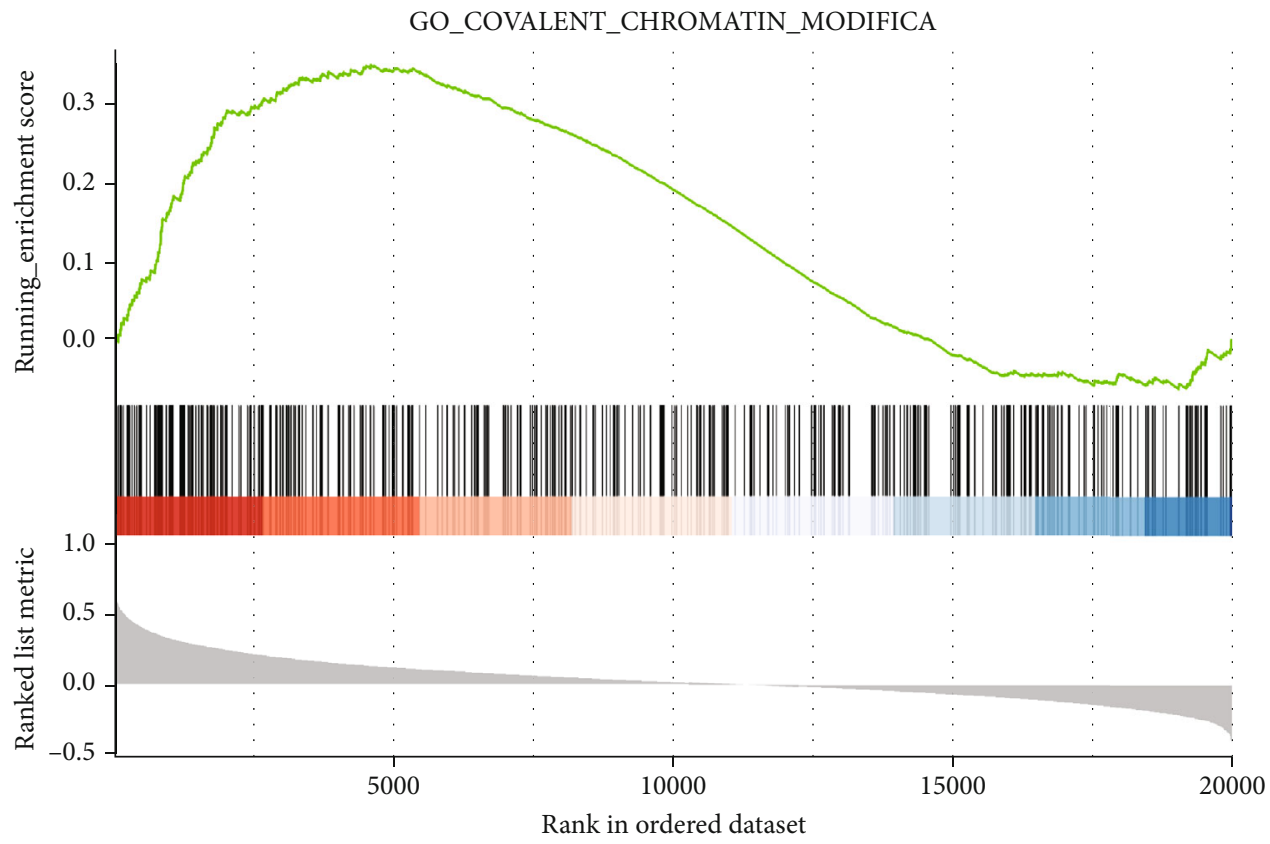

(a)

KEGG_TASTE_TRANSDUCTION

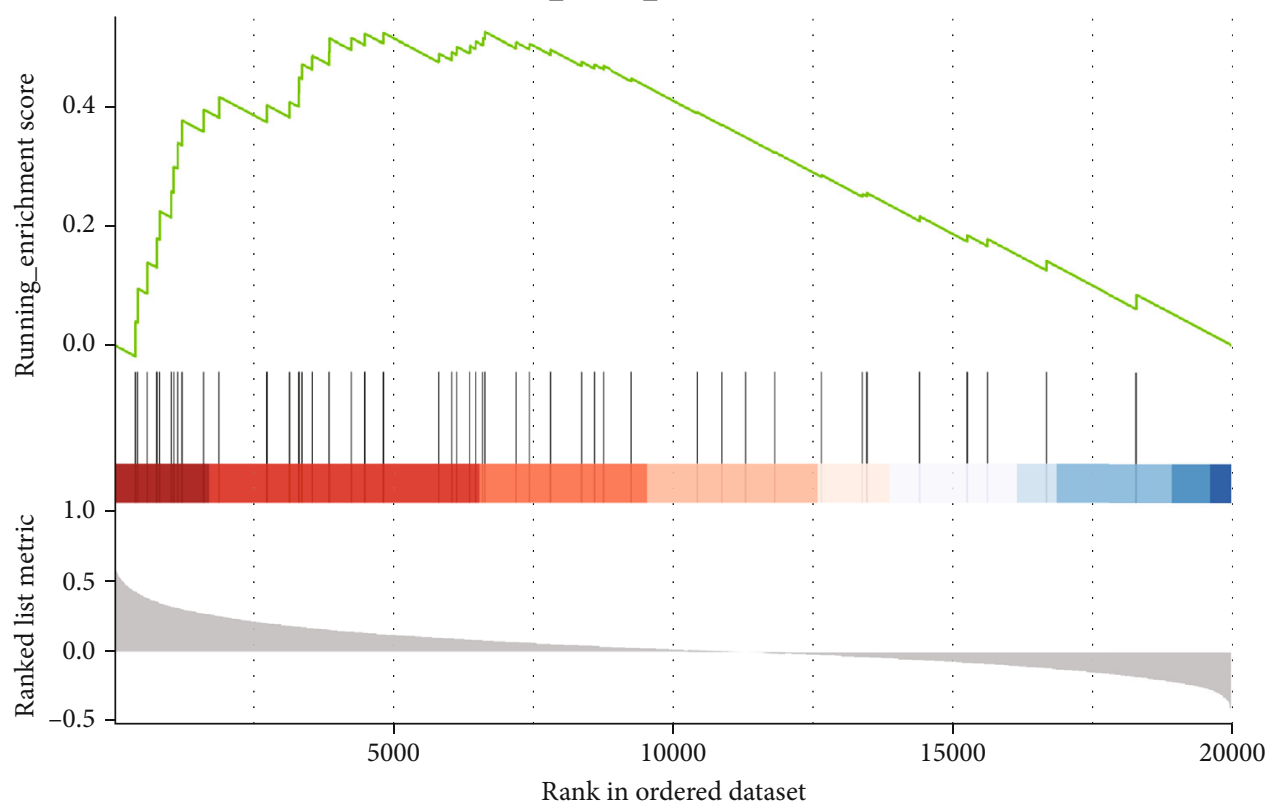

(b)

Figure 5: Continued. 


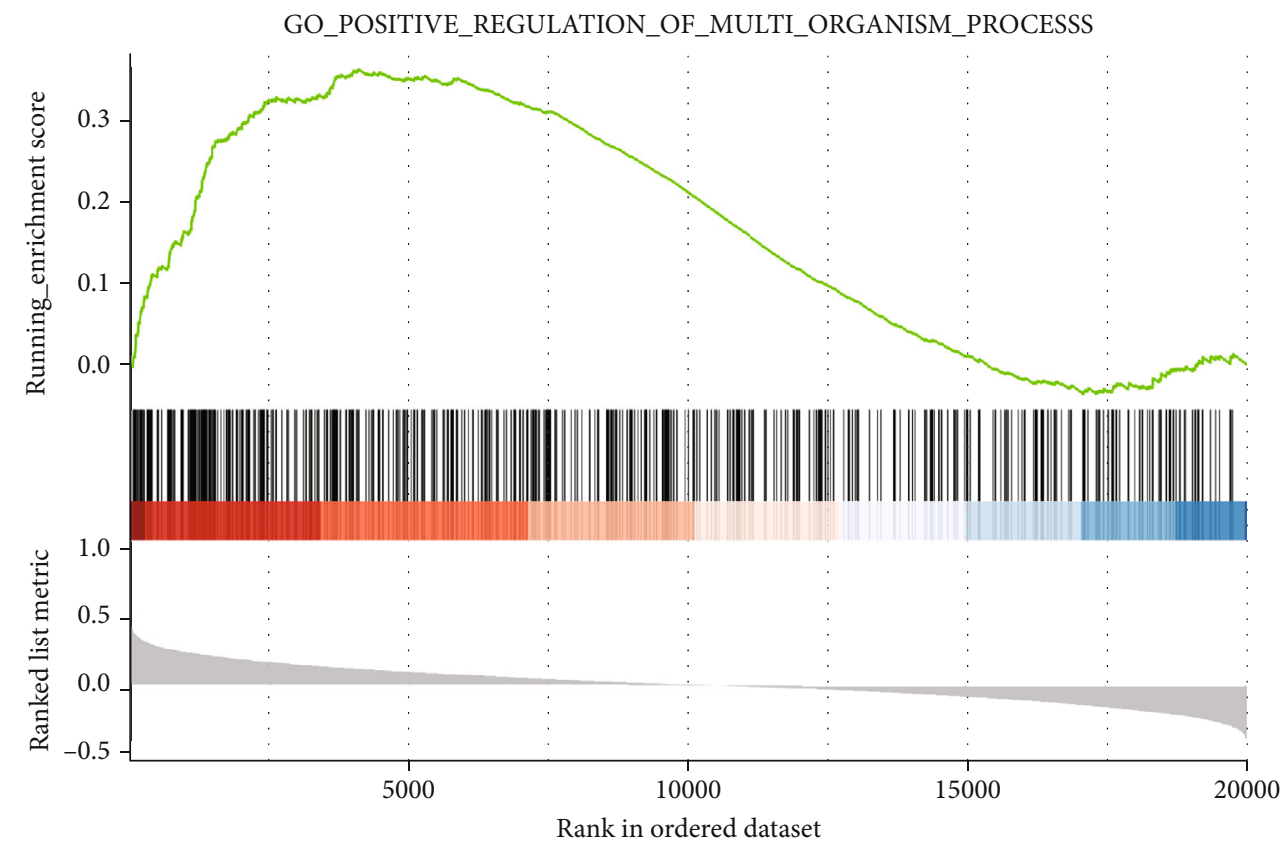

(c)

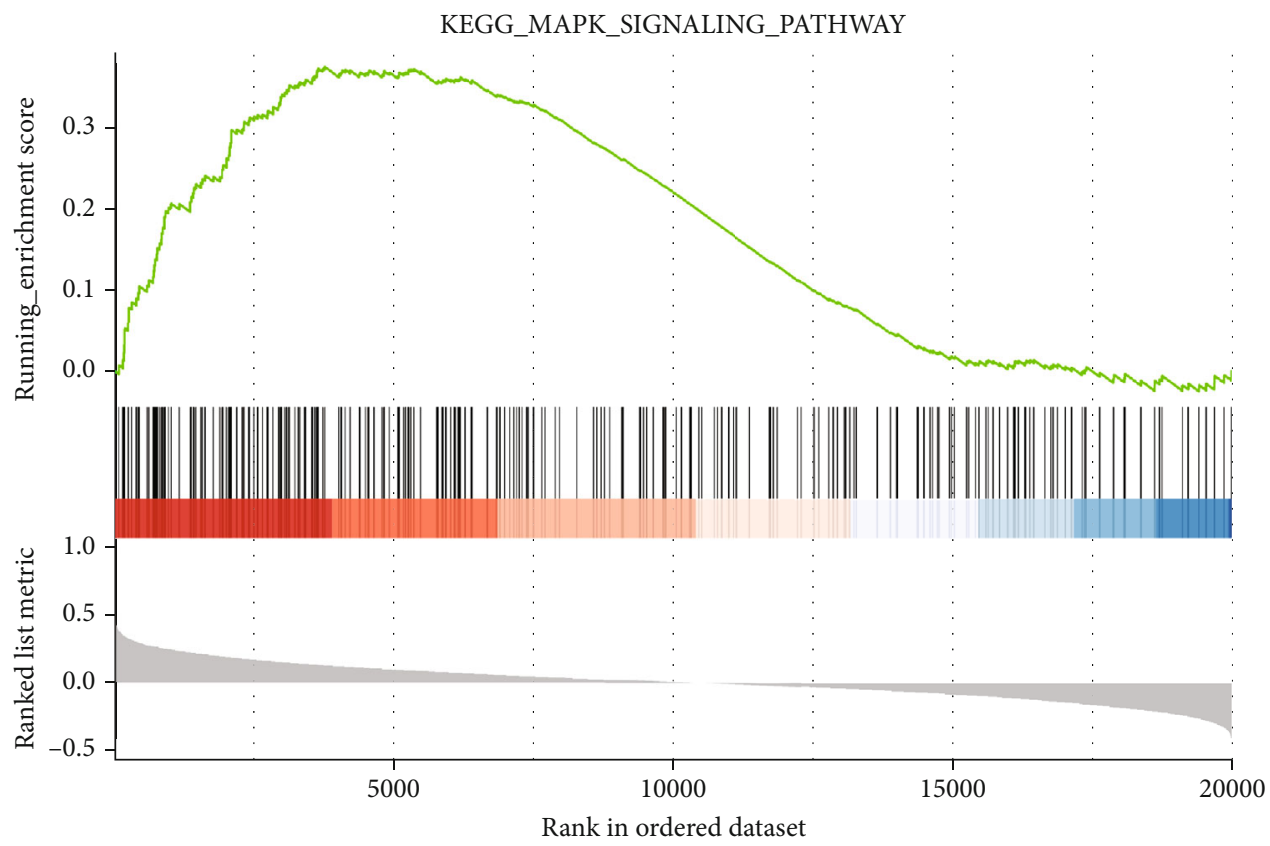

(d)

FIgUre 5: (a, b) The combination of gene ASB16 GO and KEGG results. (c, d) The combination of gene NEDD4 GO and KEGG results.

are mostly located on chromosome $5,6,15$, and 17 . In this study, the genes were ASB16 (17q21.31) and NEDD4 (15q21.3).

The protein encoded by ASB16 gene is a member of the protein family which contains the SOCS box-containing (ASB) and the repeated sequence of anchor proteins. They contain the repeat sequences of anchored protein and the SOCS box domains. Ankyrin repeat sequence is a kind of protein sequences widely existing in the organism of the dead body.

The NEDD4 gene is a founding member of the HECT ubiquitin ligase NEDD4 family, which plays a role in the protein-degrading ubiquitin proteasome system. According to a new study, the important role of the ubiquitinproteasome system also is after it is make full use of, can metabolic toxins such as garbage, fat, and cancer cells; the human body; and metabolic energy can stimulate cell reproducing itself in order to complete the self-metabolism of the human body repair function.

In this study, we identified two prognostic genes associated with LUAD survival, and it provided a basis for improving the survival rate of LUAD. Although the gene ASB18 has not been determined to be associated with the prognosis of 
LUAD, it has been shown that it is related to smoking. Smoking is an environmental risk factor for LUAD, which can be further studied.

\section{Conclusion}

Our study identified several genes that may be associated with the survival of lung adenocarcinoma, in particular two new genes (ASB16, NEDD4)) that provide evidence for the prognosis of lung adenocarcinoma, and further studies are needed to confirm our findings.

\section{Data Availability}

The datasets analyzed during the current study are obtained from UCSC Xena (https://xenabrowser.net/).

\section{Conflicts of Interest}

The authors declare that they have no conflicts of interest.

\section{Authors' Contributions}

ZS designed the study, conducted statistical analysis, interpreted the results, and wrote the initial draft of the manuscript. $\mathrm{CH}$ and $\mathrm{HC}$ contributed to the data analysis. LX, YJ, and $\mathrm{SH}$ revised the paper. All authors read and approved the final version manuscript.

\section{Acknowledgments}

The authors acknowledge the contributions of TCGA Research Network for the public availability of the cancer datasets used in our paper. This study was financially supported by a research fund from the Xuzhou Science and Technology Project (KC19017).

\section{References}

[1] F. Bray, J. Ferlay, I. Soerjomataram, R. L. Siegel, L. A. Torre, and A. Jemal, "Global cancer statistics 2018: GLOBOCAN estimates of incidence and mortality worldwide for 36 cancers in 185 countries," CA: a Cancer Journal for Clinicians, vol. 68, no. 6, pp. 394-424, 2018.

[2] Y. Yu and X. Tian, "Analysis of genes associated with prognosis of lung adenocarcinoma based on GEO and TCGA databases," Medicine, vol. 99, no. 19, article e20183, 2020.

[3] M. Dias, R. Linhas, S. Campainha, S. Conde, and A. Barroso, "Lung cancer in never-smokers - what are the differences?," Acta Oncologica, vol. 56, no. 7, pp. 931-935, 2017.

[4] J. Dai, J. Lv, M. Zhu et al., "Identification of risk loci and a polygenic risk score for lung cancer: a large-scale prospective cohort study in Chinese populations," The Lancet Respiratory Medicine, vol. 7, no. 10, pp. 881-891, 2019.

[5] T. Guo, H. Ma, and Y. Zhou, "Bioinformatics analysis of microarray data to identify the candidate biomarkers of lung adenocarcinoma," PeerJ, vol. 7, article e7313, 2019.

[6] L. Wang, X. Luo, C. Cheng, C. I. Amos, G. Cai, and F. Xiao, “A gene expression-based immune signature for lung adenocarcinoma prognosis," Cancer Immunology, Immunotherapy, vol. 69, no. 9, pp. 1881-1890, 2020.
[7] Y. Liao, Y. Wang, M. Cheng, C. Huang, and X. Fan, "Weighted gene coexpression network analysis of features that control cancer stem cells reveals prognostic biomarkers in lung adenocarcinoma," Frontiers in Genetics, vol. 11, p. 311, 2020.

[8] Y. Dong, Y. Liu, H. Bai, and S. Jiao, "Systematic assessment of the clinicopathological prognostic significance of tissue cytokine expression for lung adenocarcinoma based on integrative analysis of TCGA data," Scientific Reports, vol. 9, no. 1, p. 6301, 2019.

[9] Y. Liao, H. Xiao, M. Cheng, and X. Fan, "Bioinformatics analysis reveals biomarkers with cancer stem cell characteristics in lung squamous cell carcinoma," Frontiers in Genetics, vol. 11, p. 427, 2020.

[10] P. Zeng, X. Zhou, and S. Huang, "Prediction of gene expression with cis-SNPs using mixed models and regularization methods," BMC Genomics, vol. 18, no. 1, p. 368, 2017.

[11] C. I. Amos, X. Wu, P. Broderick et al., "Genome-wide association scan of tag SNPs identifies a susceptibility locus for lung cancer at 15q25.1," Nature Genetics, vol. 40, no. 5, pp. 616622, 2008.

[12] R. J. Hung, J. D. McKay, V. Gaborieau et al., “A susceptibility locus for lung cancer maps to nicotinic acetylcholine receptor subunit genes on 15q25," Nature, vol. 452, no. 7187, pp. 633637, 2008.

[13] P. Liu, H. G. Vikis, D. Wang et al., "Familial aggregation of common sequence variants on 15q24-25.1 in lung cancer," Journal of the National Cancer Institute, vol. 100, no. 18, pp. 1326-1330, 2008.

[14] Y. Wang, P. Broderick, E. Webb et al., "Common 5p15.33 and 6 21.33 variants influence lung cancer risk," Nature Genetics, vol. 40, no. 12, pp. 1407-1409, 2008.

[15] J. D. McKay, R. J. Hung, V. Gaborieau et al., "Lung cancer susceptibility locus at 5p15.33," Nature Genetics, vol. 40, no. 12, pp. 1404-1406, 2008.

[16] P. Broderick, Y. Wang, J. Vijayakrishnan et al., "Deciphering the impact of common genetic variation on lung cancer risk: a genome-wide association study," Cancer Research, vol. 69, no. 16, pp. 6633-6641, 2009.

[17] M. T. Landi, N. Chatterjee, K. Yu et al., "A genome-wide association study of lung cancer identifies a region of chromosome 5 p15 associated with risk for adenocarcinoma," American Journal of Human Genetics, vol. 85, no. 5, pp. 679-691, 2009.

[18] Y. Li, C. C. Sheu, Y. Ye et al., "Genetic variants and risk of lung cancer in never smokers: a genome-wide association study," The Lancet Oncology, vol. 11, no. 4, pp. 321-330, 2010.

[19] C. A. Hsiung, Q. Lan, Y. C. Hong et al., "The 5p15.33 locus is associated with risk of lung adenocarcinoma in never-smoking females in Asia," PLoS Genetics, vol. 6, no. 8, p. e1001051, 2010.

[20] D. Miki, M. Kubo, A. Takahashi et al., "Variation in TP63 is associated with lung adenocarcinoma susceptibility in Japanese and Korean populations," Nature Genetics, vol. 42, no. 10, pp. 893-896, 2010.

[21] K. A. Yoon, J. H. Park, J. Han et al., “A genome-wide association study reveals susceptibility variants for non-small cell lung cancer in the Korean population," Human Molecular Genetics, vol. 19, no. 24, pp. 4948-4954, 2010.

[22] Z. Hu, C. Wu, Y. Shi et al., "A genome-wide association study identifies two new lung cancer susceptibility loci at 13q12.12 and 22q12.2 in Han Chinese," Nature Genetics, vol. 43, no. 8, pp. 792-796, 2011. 
[23] M. J. Ahn, H. H. Won, J. Lee et al., "The 18p11.22 locus is associated with never smoker non-small cell lung cancer susceptibility in Korean populations," Human Genetics, vol. 131, no. 3, pp. 365-372, 2012.

[24] K. Shiraishi, H. Kunitoh, Y. Daigo et al., "A genome-wide association study identifies two new susceptibility loci for lung adenocarcinoma in the Japanese population," Nature Genetics, vol. 44, no. 8, pp. 900-903, 2012.

[25] M. N. Timofeeva, R. J. Hung, T. Rafnar et al., "Influence of common genetic variation on lung cancer risk: meta-analysis of 14900 cases and 29485 controls," Human Molecular Genetics, vol. 21, no. 22, pp. 4980-4995, 2012.

[26] Q. Lan, C. A. Hsiung, K. Matsuo et al., "Genome-wide association analysis identifies new lung cancer susceptibility loci in never-smoking women in Asia," Nature Genetics, vol. 44, no. 12, pp. 1330-1335, 2012.

[27] M. Chu, R. Zhang, Y. Zhao et al., "A genome-wide gene-gene interaction analysis identifies an epistatic gene pair for lung cancer susceptibility in Han Chinese," Carcinogenesis, vol. 35, no. 3, pp. 572-577, 2014.

[28] R. Zhang, M. Chu, Y. Zhao et al., "A genome-wide geneenvironment interaction analysis for tobacco smoke and lung cancer susceptibility," Carcinogenesis, vol. 35, no. 7, pp. 1528-1535, 2014.

[29] Y. Wang, J. D. McKay, T. Rafnar et al., "Rare variants of large effect in BRCA2 and CHEK2 affect risk of lung cancer," Nature Genetics, vol. 46, no. 7, pp. 736-741, 2014.

[30] S. Tang, Y. Pan, Y. Wang et al., "Genome-wide association study of survival in early-stage non-small cell lung cancer," Annals of Surgical Oncology, vol. 22, no. 2, pp. 630-635, 2015.

[31] K. A. Zanetti, Z. Wang, M. Aldrich et al., "Genome-wide association study confirms lung cancer susceptibility loci on chromosomes $5 \mathrm{p} 15$ and $15 \mathrm{q} 25$ in an African-American population," Lung Cancer, vol. 98, pp. 33-42, 2016.

[32] K. Shiraishi, Y. Okada, A. Takahashi et al., "Association of variations in HLA class II and other loci with susceptibility to EGFR-mutated lung adenocarcinoma," Nature Communications, vol. 7, no. 1, 2016.

[33] SpiroMeta Consortium, J. D. McKay, R. J. Hung et al., "Largescale association analysis identifies new lung cancer susceptibility loci and heterogeneity in genetic susceptibility across histological subtypes," Nature Genetics, vol. 49, no. 7, pp. 11261132, 2017.

[34] J. Byun, A. G. Schwartz, C. Lusk et al., "Genome-wide association study of familial lung cancer," Carcinogenesis, vol. 39, no. 9, pp. 1135-1140, 2018.

[35] X. Ji, Y. Bossé, M. T. Landi et al., "Identification of susceptibility pathways for the role of chromosome 15 q25.1 in modifying lung cancer risk," Nature Communications, vol. 9, no. 1, p. 3221, 2018.

[36] R. Li, Y. H. Yin, J. Jin et al., "Integrative analysis of DNA methylation-driven genes for the prognosis of lung squamous cell carcinoma using MethylMix," International Journal of Medical Sciences, vol. 17, no. 6, pp. 773-786, 2020.

[37] S. Dong, W. Men, S. Yang, and S. Xu, "Identification of lung adenocarcinoma biomarkers based on bioinformatic analysis and human samples," Oncology Reports, vol. 43, no. 5, pp. 1437-1450, 2020.
[38] L. M. Pardo, W. Q. Li, S. J. Hwang et al., "Genome-wide association studies of multiple keratinocyte cancers," PLoS One, vol. 12, no. 1, article e0169873, 2017.

[39] 23andMe Research Team, HUNT All-In Psychiatry, M. Liu et al., "Association studies of up to 1.2 million individuals yield new insights into the genetic etiology of tobacco and alcohol use," Nature Genetics, vol. 51, no. 2, pp. 237-244, 2019. 